\title{
Solving FIDEs by Using Semi-Analytical Techniques
}

\author{
Ahmed A. Hamoud ${ }^{1 *}$ Nedal M. Mohammed ${ }^{2}$, Kirtiwant P. Ghadle ${ }^{3}$
}

\begin{abstract}
This paper mainly focuses on the recent advances in the semi-analytical approximated methods for solving Fredholm Integro-Differential Equations (FIDEs) of the second kind by using Variational Iteration Method (VIM), Homotopy Perturbation Method (HPM) and Direct Homotopy Analysis Method (DHAM). Convergence analysis of the exact solution of the proposed methods is established. Moreover, we proved the uniqueness of the solution. To illustrate the methods, an example is presented.
\end{abstract}

Keywords: DHAM, FIDE, HPM, VIM

2010 AMS: Primary 35A15, Secondary 47G20, 65H20

${ }^{1}$ Department of Mathematics, Taiz University, Taiz, Yemen, ORCID: 0000-0002-8877-7337

${ }^{2}$ Department of Computer Science, Dr. Babasaheb Ambedkar Marathwada University, Aurangabad-India, ORCID: 0000-0002-9997-7297

${ }^{3}$ Department of Mathematics, Dr. Babasaheb Ambedkar Marathwada University, Aurangabad- India, ORCID: 0000-0003-3205-5498

*Corresponding author: drahmed985@yahoo.com

Received: 1 May 2019, Accepted: 26 July 2019, Available online: 30 September 2019

\section{Introduction}

In this paper, we consider FIDE of the form:

$$
\sum_{j=0}^{k} p_{j}(x) \Delta^{(j)}(x)=f(x)+\lambda \int_{a}^{b} W(x, t) G(\Delta(t)) d t
$$

with the initial conditions

$$
\Delta^{(r)}(a)=b_{r}, \quad r=0,1,2, \cdots,(k-1),
$$

where $\Delta^{(j)}(x)$ is the $j^{t h}$ derivative of the unknown function $\Delta(x)$ that will be determined, $W(x, t)$ is the kernel of the equation, $f(x)$ and $p_{j}(x)$ are analytic functions, $G$ is nonlinear function of $\Delta$ and $a, b, \lambda$, and $b_{r}$ are real finite constants.

The FIDEs arise in many scientific applications. It was also shown that these equations can be derived from boundary value problems.

The application of homotopy techniques in linear and non-linear problems has been devoted by scientists and engineers, because this method is to continuously deform a simple problem which is easy to solve into the under study problem which is difficult to solve. This method was proposed first by He in 1997 and systematical description in 2000 which is, in fact, a coupling of the traditional perturbation method and homotopy in topology [1]. This method was further developed and improved by He and applied to non-linear oscillators with discontinuities [2]. After that many researchers applied the method to various linear and non-linear problems. For example, it was applied to the quadratic Ricatti differential equation by Abbasbandy [3], to the axisymmetric flow over a stretching sheet by Ariel et al. [4], to the Helmholtz equation and fifth-order KdV equation by Rafei and Ganji [5], for the thin film flow of a fourth grade fluid down a vertical cylinder by Siddiqui et al. [6], to the 
non-linear Volterra-Fredholm integral equations by Hamoud and Ghadle [7], to FIDE [8], to system of Fredholm integral equations [9], Alao et al. [10] studied the ADM and the VIM on various types of integro-differential equation. Moreover, many methods for solving integro-differential equations have been studied by several authors [11,12, 13, 14, 15, 16, 17, 18, 19, 20, 21].

The main objective of the present paper is to study the behavior of the solution that can be formally determined by semi-analytical approximated methods as the VIM, HPM and DHAM. Moreover, we proved the existence and uniqueness results of the FIDEs.

\section{Variational iteration method (VIM)}

The main idea of this method is to construct a correction functional form using general Lagrange multipliers. To illustrate, we consider the following general differential equation:

$$
L \Delta(t)+N \Delta(t)=f(t),
$$

where $L$ is a linear operator, $N$ is a nonlinear operator and $f(t)$ is inhomogeneous term. According to variational iteration method [7], the terms of a sequence $\Delta_{n}$ are constructed such that this sequence converges to the exact solution. The terms $\Delta_{n}$ are calculated by a correction functional as follows:

$$
\Delta_{n+1}(t)=\Delta_{n}(t)+\int_{0}^{t} \mu(\tau)\left(L \Delta_{n}(\tau)+N \tilde{y}(\tau)-f(\tau)\right) d \tau .
$$

The successive approximation $\Delta_{n}(t), n \geq 0$ of the solution $\Delta(t)$ will be readily obtained upon using the obtained Lagrange multiplier and by using any selective function $\Delta_{0}$. The zeroth approximation $\Delta_{0}$ may be selected using any function that just satisfies at least the initial and boundary conditions. With $\mu$ determined, several approximations $\Delta_{n}(t), n \geq 0$ follow immediately.

The VIM has been shown to solve effectively, easily and accurately a large class of nonlinear problems with approximations converging rapidly to accurate solutions.

To obtain the approximation solution of IVP $(1.1)$ - (1.2), according to the VIM, the iteration formula (2.1) can be written as follows:

$$
\Delta_{n+1}(x)=\Delta_{n}(x)+L^{-1}\left[\mu(x)\left[\sum_{j=0}^{k} p_{j}(x) \Delta_{n}^{(j)}(x)-f(x)-\lambda \int_{a}^{b} W(x, t) G\left(\Delta_{n}(t)\right) d t\right]\right],
$$

where $L^{-1}$ is the multiple integration operator given as follows:

$$
L^{-1}(\cdot)=\int_{a}^{x} \int_{a}^{x} \cdots \int_{a}^{x}(\cdot) d x d x \cdots d x \quad(k-\text { times }) .
$$

To find the optimal $\mu(x)$, we proceed as follows:

$$
\begin{aligned}
\delta \Delta_{n+1}(x) & =\delta \Delta_{n}(x)+\delta L^{-1}\left[\mu(x)\left[\sum_{j=0}^{k} p_{j}(x) \Delta_{n}^{(j)}(x)-f(x)-\lambda \int_{a}^{b} W(x, t) G\left(\Delta_{n}(t)\right) d t\right]\right] \\
& =\delta \Delta_{n}(x)+\mu(x) \delta \Delta_{n}(x)-L^{-1}\left[\delta \Delta_{n}(x) \mu^{\prime}(x)\right] .
\end{aligned}
$$

From Eq. (2.2), the stationary conditions can be obtained as follows:

$$
\mu^{\prime}(x)=0 \text {, and } 1+\left.\mu(x)\right|_{x=t}=0 .
$$

As a result, the Lagrange multipliers can be identified as $\mu(x)=-1$ and by substituting in Eq. (2.2), the following iteration formula is obtained:

$$
\begin{aligned}
& \Delta_{0}(x)=L^{-1}\left[\frac{f(x)}{p_{k}(x)}\right]+\sum_{r=0}^{k-1} \frac{(x-a)^{r}}{r !} b_{r}, \\
& \Delta_{n+1}(x)=\Delta_{n}(x)-L^{-1}\left[\sum_{j=0}^{k} p_{j}(x) \Delta_{n}^{(j)}(x)-f(x)-\lambda \int_{a}^{b} W(x, t) G\left(\Delta_{n}(t)\right) d t\right], n \geq 0 .
\end{aligned}
$$

The term $\sum_{r=0}^{k-1} \frac{(x-a)^{r}}{r !} b_{r}$ is obtained from the initial conditions, $p_{k}(x) \neq 0$. Relation (2.3) will enable us to determine the components $\Delta_{n}(x)$ recursively for $n \geq 0$. Consequently, the approximation solution may be obtained by using

$$
\Delta(x)=\lim _{n \rightarrow \infty} \Delta_{n}(x) .
$$




\section{Homotopy perturbation method (HPM)}

The homotopy perturbation method first proposed by He [1, 2]. To illustrate the basic idea of this method, we consider the following nonlinear differential equation

$$
A(\Delta)-f(r)=0, \quad r \in \Omega,
$$

under the boundary conditions

$$
B\left(\Delta, \frac{\partial \Delta}{\partial n}\right)=0, \quad r \in \Gamma,
$$

where $A$ is a general differential operator, $B$ is a boundary operator, $f(r)$ is a known analytic function, $\Gamma$ is the boundary of the domain $\Omega$.

In general, the operator $A$ can be divided into two parts $L$ and $N$, where $L$ is linear, while $N$ is nonlinear. Eq. (3.1) therefore can be rewritten as follows [19]:

$$
L(\Delta)+N(\Delta)-f(r)=0 .
$$

By the homotopy technique, we will construct a homotopy $v(r, p): \Omega \times[0,1] \longrightarrow \mathbb{R}$ which satisfies

$$
H(v, p)=(1-p)\left[L(v)-L\left(\Delta_{0}\right)\right]+p[A(v)-f(r)]=0, p \in[0,1] .
$$

or

$$
\left.H(v, p)=L(v)-L\left(\Delta_{0}\right)+p L\left(\Delta_{0}\right)\right]+p[N(v)-f(r)]=0,
$$

where $p \in[0,1]$ is an embedding parameter, $\Delta_{0}$ is an initial approximation of Eq.(3.1) which satisfies the boundary conditions. From Eqs.(3.2), (3.3) we have

$$
\begin{aligned}
& H(v, 0)=L(v)-L\left(\Delta_{0}\right)=0, \\
& H(v, 1)=A(v)-f(r)=0 .
\end{aligned}
$$

The changing in the process of $p$ from zero to unity is just that of $v(r, p)$ from $\Delta_{0}(r)$ to $\Delta(r)$. In topology this is called deformation, the $L(v)-L\left(\Delta_{0}\right)$, and $A(v)-f(r)$ are called homotopic. Now, assume that the solution of Eqs. (3.2) and (3.3) can be expressed as

$$
v=v_{0}+p v_{1}+p^{2} v_{2}+\cdots .
$$

The approximate solution of Eq.(3.1) can be obtained by setting $p=1$.

$$
\Delta=\lim _{p \rightarrow 1} v=v_{0}+v_{1}+v_{2}+\cdots .
$$

Then equating the terms with identical power of $P$, we obtain the following series of linear equations:

$$
\begin{aligned}
& P^{0}: \Delta_{0}(x)=\sum_{r=0}^{k-1} \frac{1}{r !}(x-a)^{r} b_{r}, \\
& P^{1}: \Delta_{1}(x)=L^{-1}\left(\frac{f(x)}{p_{k}(x)}\right)+\lambda L^{-1}\left(\int_{a}^{b} \frac{W(x, t)}{p_{k}(x)} G\left(\Delta_{0}(t)\right)(t) d t\right)-\sum_{j=0}^{k-1} L^{-1}\left(\frac{p_{j}(x)}{p_{k}(x)} \Delta_{0}^{(j)}(x)\right), \\
& P^{2}: \Delta_{2}(x)=\lambda L^{-1}\left(\int_{a}^{b} \frac{W(x, t)}{p_{k}(x)} G\left(\Delta_{1}(t)\right)(t) d t\right)-\sum_{j=0}^{k-1} L^{-1}\left(\frac{p_{j}(x)}{p_{k}(x)} \Delta_{1}^{(j)}(x)\right) \\
& P^{3}: \Delta_{3}(x)=\lambda L^{-1}\left(\int_{a}^{b} \frac{W(x, t)}{p_{k}(x)} G\left(\Delta_{2}(t)\right) d t\right)-\sum_{j=0}^{k-1} L^{-1}\left(\frac{p_{j}(x)}{p_{k}(x)} \Delta_{2}^{(j)}(x)\right)
\end{aligned}
$$




\section{Direct homotopy analysis method (DHAM)}

Consider FIDE (1.1) and substitute the kernel $W(x, t)=g(x) h(t)$ we obtain

$$
\sum_{j=0}^{k} p_{j}(x) \Delta^{(j)}(x)=f(x)+\lambda g(x) \int_{a}^{b} h(t) G(\Delta(t)) d t
$$

To obtain the approximate solution, we integrating $(k)$-times in the interval $[a, x]$ with respect to $x$ we obtain,

$$
\Delta(x)=L^{-1}\left(\frac{f(x)}{p_{k}(x)}\right)+\sum_{r=0}^{k-1} \frac{1}{r !}(x-a)^{r} b_{r}+\lambda L^{-1}\left(\frac{g(x)}{p_{k}(x)} \int_{a}^{b} h(t) G(\Delta(t)) d t\right)-\sum_{j=0}^{k-1} L^{-1}\left(\frac{p_{j}(x)}{p_{k}(x)} \Delta_{n}^{(j)}(x)\right),
$$

Setting

$$
\begin{aligned}
Q & =\int_{a}^{b} h(t) G(\Delta(t)) d t \\
F & =L^{-1}\left(\frac{f(x)}{p_{k}(x)}\right)+\sum_{r=0}^{k-1} \frac{1}{r !}(x-a)^{r} b_{r}-\sum_{j=0}^{k-1} L^{-1}\left(\frac{p_{j}(x)}{p_{k}(x)} \Delta_{n}^{(j)}(x)\right) .
\end{aligned}
$$

Therefore, we can rewrite Eq. (4.1) as

$$
\Delta(x)=F(x)+\lambda L^{-1}\left(\frac{g(x)}{p_{k}(x)} Q\right),
$$

we define the nonlinear homotopy operator as:

$$
N[\Delta(x)]=\Delta(x)-F(x)-\lambda L^{-1}\left(\frac{g(x)}{p_{k}(x)} Q\right),
$$

The corresponding mth-order deformation equation is as follows

$$
L\left[\Delta_{m}(x)-\chi_{m} \Delta_{m-1}(x)\right]=B H(x) R_{m}\left(\overrightarrow{\Delta_{m-1}(x)}\right)
$$

where

$$
R_{m}\left(\overrightarrow{\Delta_{m-1}(x)}\right)=\Delta_{m-1}(x)-F(x)\left(1-\chi_{m}\right)-\lambda L^{-1}\left(\frac{g(x)}{p_{k}(x)} Q\right),
$$

and

$$
\chi_{m}= \begin{cases}1, & m>1 . \\ 0, & m \leq 1 .\end{cases}
$$

choosing the auxiliary linear operator $L[\Delta]=\Delta$, we obtain

$$
\Delta_{0}(x) \quad \text { Choosing initial guess }
$$

$$
\begin{aligned}
\Delta_{1}(x)= & B H(x)\left[\Delta_{0}(x)-L^{-1}\left(\frac{f(x)}{p_{k}(x)}\right)-\sum_{r=0}^{k-1} \frac{1}{r !}(x-a)^{r} b_{r}-\lambda L^{-1}\left(\frac{g(x)}{p_{k}(x)} \int_{a}^{b} h(t) G\left(\Delta_{0}(t)\right) d t\right)\right. \\
& \left.+\sum_{j=0}^{k-1} L^{-1}\left(\frac{p_{j}(x)}{p_{k}(x)} \Delta_{0}^{(j)}(x)\right)\right], \\
\Delta_{m}(x)= & \chi_{m} \Delta_{m-1}(x)+B H(x)\left[\Delta_{m-1}(x)-\lambda L^{-1}\left(\frac{g(x)}{p_{k}(x)} \int_{a}^{b} h(t) G\left(\Delta_{m-1}(t)\right) d t\right)\right. \\
& \left.+\sum_{j=0}^{k-1} L^{-1}\left(\frac{p_{j}(x)}{p_{k}(x)} \Delta_{m-1}^{(j)}(x)\right)\right], m>1 .
\end{aligned}
$$

with auxiliary function $H(x)$ and auxiliary parameter $B$.

Then, $\Delta(x)=\sum_{i=0}^{m} \Delta_{i}$ as the approximate solution. 


\section{Uniqueness results}

In this section, we shall give an uniqueness results of Eq. (1.1), with the initial condition (1.2) and prove it [22, 23].

We can be written equation (1.1) in the form of:

$$
\Delta(x)=L^{-1}\left[\frac{f(x)}{p_{k}(x)}\right]+\sum_{r=0}^{k-1} \frac{(x-a)^{r}}{r !} b_{r}+\lambda_{1} L^{-1}\left[\int_{a}^{b} \frac{1}{p_{k}(x)} W(x, t) G\left(\Delta_{n}(t)\right) d t\right]-L^{-1}\left[\sum_{j=0}^{k-1} \frac{p_{j}(x)}{p_{k}(x)} \Delta^{(j)}(x)\right] .
$$

we can write

$$
\begin{aligned}
& L^{-1}\left[\int_{a}^{b} \frac{1}{p_{k}(x)} W(x, t) G\left(\Delta_{n}(t)\right) d t\right]=\int_{a}^{b} \frac{(x-t)^{k}}{k ! p_{k}(x)} W(x, t) G\left(\Delta_{n}(t)\right) d t \\
& \sum_{j=0}^{k-1} L^{-1}\left[\frac{p_{j}(x)}{p_{k}(x)}\right] \Delta^{(j)}(x)=\sum_{j=0}^{k-1} \int_{a}^{b} \frac{(x-t)^{k-1} p_{j}(t)}{k-1 ! p_{k}(t)} \Delta^{(j)}(t) d t .
\end{aligned}
$$

We set,

$$
\Psi(x)=L^{-1}\left[\frac{f(x)}{p_{k}(x)}\right]+\sum_{r=0}^{k-1} \frac{(x-a)^{r}}{r !} b_{r} .
$$

Before starting and proving the main results, we introduce the following hypotheses:

(H1) There exist two constants $\alpha$ and $\gamma_{j}>0, j=0,1, \cdots, k$ such that, for any $\Delta_{1}, \Delta_{2} \in C(J, \mathbb{R})$

$$
\left.\left.\mid G\left(\Delta_{1}\right)\right)-G\left(\Delta_{2}\right)\right)|\leq \alpha| \Delta_{1}-\Delta_{2} \mid
$$

and

$$
\left|D^{j}\left(\Delta_{1}\right)-D^{j}\left(\Delta_{2}\right)\right| \leq \gamma_{j}\left|\Delta_{1}-\Delta_{2}\right|
$$

we suppose that the nonlinear terms $G(\Delta(x))$ and $D^{j}(\Delta)=\left(\frac{d^{j}}{d x^{j}}\right) \Delta(x)=\sum_{i=0}^{\infty} \gamma_{i_{j}}$, ( $D^{j}$ is a derivative operator), $j=$ $0,1, \cdots, k$, are Lipschitz continuous.

(H2) We suppose that for all $a \leq t \leq x \leq b$, and $j=0,1, \cdots, k$ :

$$
\left|\frac{\lambda(x-t)^{k} W(x, t)}{k ! p_{k}(x)}\right| \leq \theta_{1}, \quad\left|\frac{\lambda(x-t)^{k} W(x, t)}{k !}\right| \leq \theta_{2},
$$

and

$$
\left|\frac{(x-t)^{k-1} p_{j}(t)}{(k-1) ! p_{k}(t)}\right| \leq \theta_{3}, \quad\left|\frac{(x-t)^{k-1} p_{j}(t)}{(k-1) !}\right| \leq \theta_{4},
$$

(H3) There exist three functions $\theta_{3}^{*}, \theta_{4}^{*}$, and $\gamma^{*} \in C\left(D, \mathbb{R}^{+}\right)$, the set of all positive function continuous on $D=\{(x, t) \in$ $\mathbb{R} \times \mathbb{R}: 0 \leq t \leq x \leq 1\}$ such that:

$$
\theta_{3}^{*}=\max \left|\theta_{3}\right|, \quad \theta_{4}^{*}=\max \left|\theta_{4}\right|, \text { and } \gamma^{*}=\max \left|\gamma_{j}\right| .
$$

(H4) $\Psi(x)$ is bounded function for all $x$ in $J=[a, b]$.

Theorem 5.1. Assume that (H1)-(H4) hold. If

$$
0<\psi=\left(\alpha \theta_{1}+k \gamma^{*} \theta_{3}^{*}\right)(b-a)<1,
$$

then there exists a unique solution $\Delta(x) \in C(J)$ to IVP $(1.1)-(1.2)$. 
Proof. Let $\Delta_{1}$ and $\Delta_{2}$ be two different solutions of IVP (1.1) - (1.2)., then

$$
\begin{aligned}
\left|\Delta_{1}-\Delta_{2}\right|= & \left.\mid \int_{a}^{b} \frac{\lambda(x-t)^{k} W(x, t)}{p_{k}(x) k !}\left[G\left(\Delta_{1}\right)-G\left(\Delta_{2}\right)\right)\right] d t \\
& \left.-\sum_{j=0}^{k-1} \int_{a}^{b} \frac{(x-t)^{k-1} p_{j}(t)}{p_{k}(t)(k-1) !}\left[D^{j}\left(\Delta_{1}\right)-D^{j}\left(\Delta_{2}\right)\right)\right] d t \mid \\
\leq & \left.\int_{a}^{b}\left|\frac{\lambda(x-t)^{k} W(x, t)}{p_{k}(x) k !}\right| \mid G\left(\Delta_{1}\right)-G\left(\Delta_{2}\right)\right) \mid d t \\
& \left.-\sum_{j=0}^{k-1} \int_{a}^{b}\left|\frac{(x-t)^{k-1} p_{j}(t)}{p_{k}(t)(k-1) !}\right| \mid D^{j}\left(\Delta_{1}\right)-D^{j}\left(\Delta_{2}\right)\right) \mid d t \\
\leq & \left(\alpha \theta_{1}+k \gamma^{*} \theta_{3}^{*}\right)(b-a)\left|\Delta_{1}-\Delta_{2}\right|,
\end{aligned}
$$

we get $(1-\psi)\left|\Delta_{1}-\Delta_{2}\right| \leq 0$. Since $0<\psi<1$, so $\left|\Delta_{1}-\Delta_{2}\right|=0$. Therefore, $\Delta_{1}=\Delta_{2}$ and the proof is completed.

\section{Example}

In this section, we present the semi-analytical techniques based on VIM, HPM and DHAM to solve FIDEs. To show the efficiency of the present methods for our problem in comparison with the exact solutions.

Example 6.1. Consider the following FIDE:

$$
\Delta^{\prime}(x)=e^{x}(1+x)-x+\int_{0}^{1} x \Delta(t) d t
$$

with the initial condition

$$
\Delta(0)=0,
$$

and the the exact solution is $\Delta(x)=x e^{x}$.

Table 1. Numerical Results of the Example 6.1

\begin{tabular}{lcccc}
\hline $\mathrm{x}$ & Exact & VIM & HPM & DHAM \\
\hline 0.1 & 0.1105170 & 0.1096837 & 0.1103782 & 0.1105170 \\
0.2 & 0.2442805 & 0.2409472 & 0.2437249 & 0.2442805 \\
0.3 & 0.4049576 & 0.3974576 & 0.4037076 & 0.4049576 \\
0.4 & 0.5967298 & 0.5833965 & 0.5945076 & 0.5967298 \\
0.5 & 0.8243606 & 0.8035273 & 0.8208884 & 0.8233606 \\
0.6 & 1.0932712 & 1.0632712 & 1.0882712 & 1.0932712 \\
0.7 & 1.4096268 & 1.3687935 & 1.4028213 & 1.4096268 \\
0.8 & 1.7804327 & 1.7270994 & 1.7715438 & 1.7804327 \\
0.9 & 2.2136428 & 2.1461428 & 2.2023928 & 2.2136428 \\
\hline
\end{tabular}

\section{Discussion and conclusion}

We discussed the VIM, HPM and DHAM for solving FIDEs of the second kind. To assess the accuracy of each method, the test example with known exact solution is used. In this work, the above methods have been successfully employed to obtain the approximate solution of a FIDE. The results show that these methods are very efficient, convenient and can be adapted to fit a larger class of problems. The comparison reveals that although the numerical results of these methods are similar approximately, Table 1 shows that the numerical results obtained with DHAM agree with the exact solutions. 


\section{References}

[1] J.H. He, A coupling method of a homotopy technique and a perturbation technique for non-linear problems, Int. J. Non-Linear Mech., 35(1) (2000), 37-43.

[2] J.H. He, The homotopy perturbation method for non-linear oscillators with discontinuities, Appl. Math. Comput., 151(1) (2004), 287-292.

[3] S. Abbasbandy, Iterated He's homotopy perturbation method for quadratic Riccati differential equation, Appl. Math. Comput., 175(1) (2006), 581-589.

[4] P. Ariel, T. Hayat, S. Asghar, Homotopy perturbation method and axisymmetric flow over a stretching sheet, Int. J. Nonlinear Sci. Numer. Simul., 7(4) (2006), 399-406.

[5] M. Rafei, D.D. Ganji, Explicit solutions of Helmholtz equation and fifth-order KdV equation using homotopy perturbation method, Int. J. Nonlinear Sci. Numer. Simul., 7(3) (2006), 321-328.

[6] A.M. Siddiqui, R. Mahmood, Q.K. Ghori, Homotopy perturbation method for thin film flow of a fourth grade fluid down a vertical cylinder, Phys. Lett. A, 352(4-5) (2006), 404-410.

[7] A. Hamoud, K. Ghadle, A study of some reliable methods for solving fuzzy Volterra-Fredholm integral equations, Acta Univ. Apul., 53 (2018), 65-92.

[8] A. Hamoud, K. Ghadle, S. Atshan The approximate solutions of fractional integro-differential equations by using modified Adomian decomposition method, Khayyam J. Math., 5(1) (2019), 21-39.

[9] M. Javidi, A Golbabai, A numerical solution for solving system of Fredholm integral equations by using homotopy perturbation method, Appl. Math. Comput., 189(2) (2007), 1921-1928.

[10] S. Alao, F. Akinboro, F. Akinpelu, R. Oderinu, Numerical solution of integro-differential equation using Adomian decomposition and variational iteration methods, IOSR Journal of Mathematics, 10(4) (2014), 18-22.

[11] A. Hamoud, K. Ghadle, The reliable modified of Laplace Adomian decomposition method to solve nonlinear interval Volterra-Fredholm integral equations, Korean J. Math. 25(3) (2017), 323-334.

[12] A. Hamoud, K. Ghadle, On the numerical solution of nonlinear Volterra-Fredholm integral equations by variational iteration method, Int. J. Adv. Sci. Tech. Research, 3 (2016), 45-51.

[13] A. Hamoud, K. Ghadle, The combined modified Laplace with Adomian decomposition method for solving the nonlinear Volterra-Fredholm integro-differential equations, J. Korean Soc. Ind. Appl. Math. 21 (2017), 17-28.

[14] A. Hamoud, K. Ghadle, Modified Adomian decomposition method for solving fuzzy Volterra-Fredholm integral equations, J. Indian Math. Soc. 85(1-2) (2018), 52-69.

[15] A. Hamoud, K. Ghadle, Recent advances on reliable methods for solving Volterra-Fredholm integral and integro-differential equations, Asian Journal of Mathematics and Computer Research, 24(3) (2018), 128-157.

[16] A. Hamoud, K. Ghadle, M. Bani Issa, Giniswamy, Existence and uniqueness theorems for fractional Volterra-Fredholm integro-differential equations, Int. J. Appl. Math., 31(3) (2018), 333-348.

[17] A. Hamoud, K. Ghadle, Existence and uniqueness of the solution for Volterra-Fredholm integro-differential equations, Journal of Siberian Federal University. Mathematics \& Physics, 11(6) (2018), 692-701.

[18] A. Hamoud, A. Azeez, K. Ghadle, A study of some iterative methods for solving fuzzy Volterra-Fredholm integral equations, Indonesian J. Elec. Eng. \& Comp. Sci., 11(3) (2018), 1228-1235.

[19] A. Hamoud, K. Ghadle, Homotopy analysis method for the first order fuzzy Volterra-Fredholm integro-differential equations, Indonesian J. Elec. Eng. \& Comp. Sci., 11(3) (2018), 857-867.

[20] A. Hamoud, K. Ghadle, Usage of the homotopy analysis method for solving fractional Volterra-Fredholm integrodifferential equation of the second kind, Tamkang Journal of Mathematics, 49(4) (2018), 301-315.

[21] A. Hamoud, M. Bani Issa, K. Ghadle, M. Abdulghani, Existence and convergence results for Caputo fractional Volterra integro-differential equations, Journal of Mathematics and Applications, 41 (2018), 109-122.

[22] A. Hamoud, K. Ghadle, Existence and uniqueness of solutions for fractional mixed Volterra-Fredholm integro-differential equations, Indian J. Math., 60(3) (2018), 375-395.

[23] A. Hamoud, K. Ghadle, Modified Laplace decomposition method for fractional Volterra-Fredholm integro-differential equations, Journal of Mathematical Modeling, 6(1) (2018), 91-104. 